\title{
Phase diagram for a Bose-Einstein condensate moving in an optical lattice
}

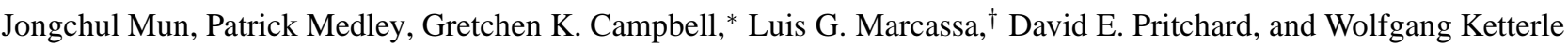 \\ MIT-Harvard Center for Ultracold Atoms, Research Laboratory of Electronics, \\ and Department of Physics, MIT, Cambridge, Massachusetts 02139, USA.
}

(Dated: October 31, 2018)

\begin{abstract}
The stability of superfluid currents in a system of ultracold bosons was studied using a moving optical lattice. Superfluid currents in a very weak lattice become unstable when their momentum exceeds 0.5 recoil momentum. Superfluidity vanishes already for zero momentum as the lattice deep reaches the Mott insulator(MI) phase transition. We study the phase diagram for the disappearance of superfluidity as a function of momentum and lattice depth between these two limits. Our phase boundary extrapolates to the critical lattice depth for the superfluid-to-MI transition with $2 \%$ precision. When a one-dimensional gas was loaded into a moving optical lattice a sudden broadening of the transition between stable and unstable phases was observed.
\end{abstract}

PACS numbers: 03.75.Lm,03.75.Kk

The realization of condensed matter systems using ultracold atoms brings the precision and control of atomic physics to the study of many-body physics. Many studies have focussed on Mott insulator physics, an important paradigm for the suppression of transport by particle correlations. Previous studies of the superfluid(SF)-to-Mott insulator(MI) transition in optical lattices with ultracold bosons [1, 2, 3, 4, 5, 6, 7, 8] adressed the quenching of superfluidity below a critical lattice depth. Here we extend these studies into a second dimension by studying stability of superfluid current as a function of momentum and lattice depth as suggested in ref. [9]. These transport measurements show the stability of the quantum phase in a moving system, which is far from equilibrium.

Transport measurements extend previous work on stationary systems in two regards. First, superfluidity near the MI transition has only been indirectly inferred from coherence measurements, whereas in this work, we characterize the superfluid regime by observing a critical current for superfluid flow. Second, previous studies $[1,2,3,4,6,6,6,8]$ were not able to precisely locate the phase transition, since the observed excitation spectrum and atomic interference pattern did not abruptly change [3, 5, 6], partially due to the inhomogeneous density. In contrast, the sudden onset of dissipation provides a clear distinction between the two quantum phases. In the SF phase, current flows without dissipation if the momentum does not exceed a critical momentum, while in the MI phase the critical momentum vanishes and transport is dissipative.

Bosonic atoms in an optical lattice are often described by the Bose-Hubbard Model where the tunneling between nearest neighbour lattice sites is characterized by the hopping matrix element $J$ and the repulsive interactions by the on-site matrix element $U[1,10,11,12]$. The dimensionless interaction energy $u \equiv U / J$ determines the quantum phase of the system. For $u>u_{c}$, the system is in the MI phase; for $u<u_{c}$, it is in the SF phase.

For weak interactions $(u \rightarrow 0)$, the system approaches single-particle physics in a periodic potential well described by Bloch states and band structure. The critical momentum for a stable current-carrying state is $0.5 p_{r}\left(p_{r}=h / \lambda\right.$ is the recoil momentum of an atom, where $\lambda$ is the wavelength of

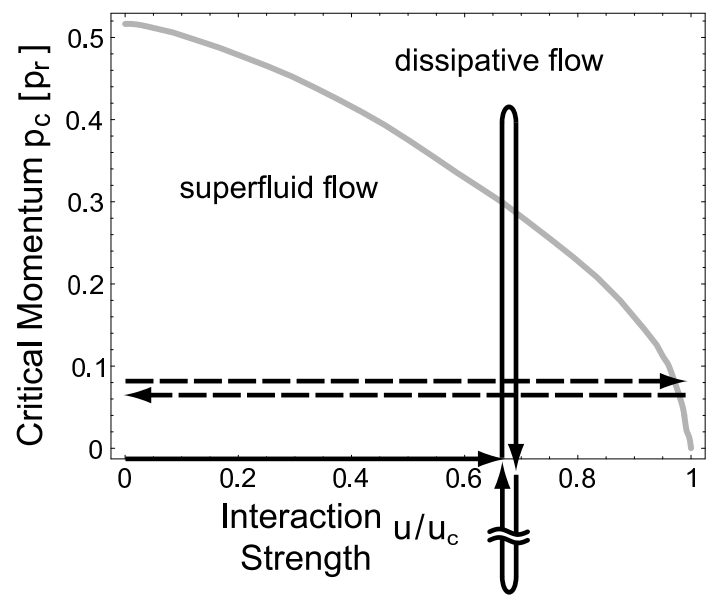

FIG. 1: Phase diagram showing the stability of superfluid flow in an optical lattice and the experimental procedure. The grey curve shows the predicted boundary between superfluid flow and dissipative flow phases for a three-dimensional gas with a commensurate filling of $N=1$ atom per site [9]. The solid (dashed) arrows illustrate the experimental trajectory used for small (large) lattice depths (see text for details).

the optical lattice light) [13]. At the critical momentum, it becomes possible for two atoms in the same initial Bloch state to scatter into two other states and conserve energy and quasimomentum [14, 15]. For sufficiently deep lattices this occurs when the effective mass becomes negative. Instabilities in a 1D optical lattice were studied theoretically using a linear stability analysis of the Gross-Pitaevskii Equation [13, 16] , and experimentally [7, 17]. The theoretical studies predicted that for increasing lattice depth or increasing atomic interactions the stability of superfluid flow should increase [13, 16]: the dynamic instability would stay near $0.5 p_{r}$ whereas the Landau critical velocity and therefore the energetic instability would shift to larger momenta (For more discussions on dynamic and energetic instability, see ref. [18, 19]). However, these analyses neglect the growing importance of quantum correlations for larger lattice depth which lead to the SF-MI phase transition, where the critical momentum for a superfluid 
current vanishes. In this paper, we study the decrease of the critical momentum from its value for the weakly interacting regime towards zero at the MI transition.(Fig. (1)

Most studies of the SF-MI phase transition monitor the coherence in the superfluid phase through an interference pattern observed in the ballistic expansion resulting from a sudden turn-off of the confining potential and lattice. Previous observations of the phase transition found the experimental transition point to lie in the range between 10 and $13 E_{R}$ (with the recoil energy defined as $E_{R}=p_{r}^{2} / 2 m$ where $m$ is the atomic mass) [3]. This uncertainty is related to the inhomogeneous density profile of trapped atoms and to the fact that the visibility of the interference extends beyond the transition point due to short-range coherence in the MI phase [6]. It has been suggested that observed kinks in the visibility are linked to the formation of the MI shells with occupation numbers $N=2$ and 3 [6]. Several authors have suggested other features in the momentum distribution beyond coherent interference peaks as a more distinct signature of the phase transition [20, 21]. Here we show that the disappearance of the critical momentum for superfluid flow provides such a signature and allows the determination of the transition point with high precision.

Our measurement was not limited by the inhomogeneous density profile. For our range of lattice depths, low critical momenta and the onset of dissipation occur only near the formation of MI shells with integer occupation numbers $N$ [9]. The onset of dissipation related to the $N=1$ domains occurs at smaller momentum than for other $N$ domains. For instance, with increasing momentum $p$ the $N=1$ domain becomes unstable first, and this triggers dissipation over the whole atomic cloud [9]. Therefore, the breakdown of superfluid flow in the system was determined by the formation of the $N=1$ domain and was not smeared out by the inhomogeneous density. Our criterion, the sudden onset of dissipation, depended on the formation of an insulating shell surrounded by a superfluid region, which occurs only in the inhomogeneous case.

In our experimental setup, a Bose-Einstein condensate of ${ }^{87} \mathrm{Rb}$ atoms in the $5 \mathrm{~S}_{1 / 2}|1,-1\rangle$ state was prepared and trapped in a combination of an Ioffe-Pritchard magnetic trap and an optical dipole trap. The number of atoms in the BEC was typically $2 \times 10^{5}$, resulting in a maximum filling factor $N$ of around 3. The magnetic trap frequencies were $\omega_{x, y}=40 \mathrm{~Hz}$ radially and $\omega_{z}=4.6 \mathrm{~Hz}$ axially. The laser beam for the optical dipole trap was oriented along the $\mathrm{x}$ axis. This laser beam was retroreflected and the polarization of the retroreflected beam was rotated in order to minimize interference between the two beams. Along the vertical direction (y-axis) a lattice was formed by a retroreflected laser beam. For the z-axis, a moving lattice was created by introducing a small frequency detuning $\delta f$ between the two counter-propagating laser beams using acousto-optical modulators driven by phase-locked frequency generators. The 3D optical lattice was ramped up in the following way: For the lattice along the $\mathrm{x}$-axis, the polarization of the retroreflected beam was rotated to increase the interference contrast. For the other two axes, the power was increased exponentially in 160 (a)
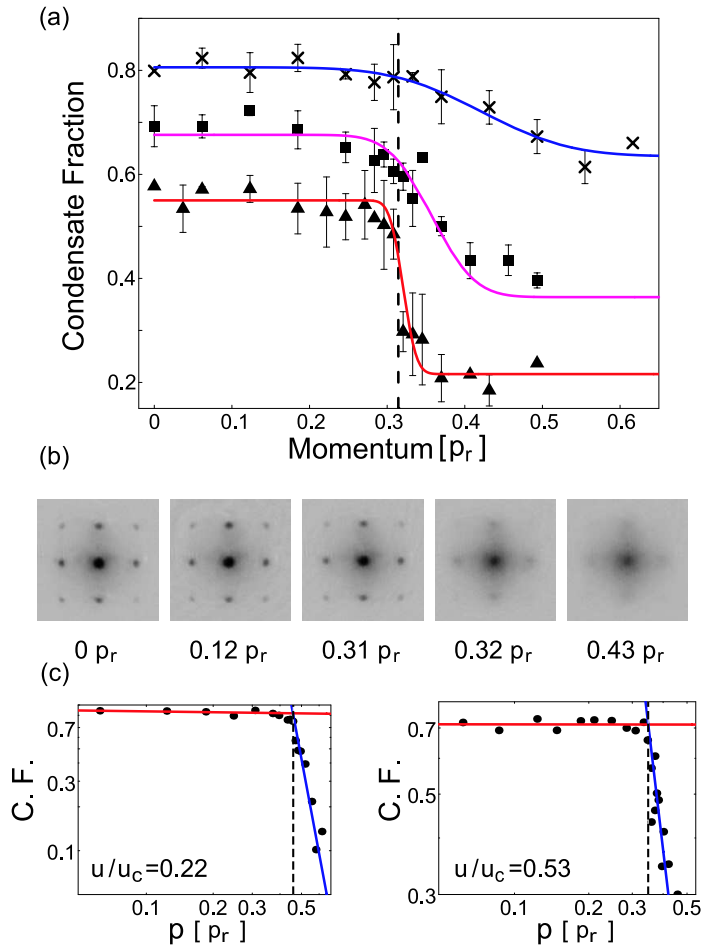

FIG. 2: Determination of the critical momentum of superfluid flow. Shown is the condensate fraction as a function of a momentum $p$. (a) Condensate fraction with $u / u_{c}=0.61$ for a variable number of cycles of the momentum modulation (one cycle: cross marks and blue line, two cycles: squares and purple line, three cycles: triangles and red line). A dashed vertical line indicates the critical momentum where instability begins to occur. The two and three-cycle data are offset vertically for clarity. These data were fitted with an error function to guide the eye. (b) Images of interference patterns released from an optical lattice at $u / u_{c}=0.61$ moving with variable momentum. Instability occurred between $p=0.31 p_{r}$ and $0.32 p_{r}$. Some of the triangular data points in (a) were obtained from these images. (c) Condensate fraction on a log-log scale for two different interaction strengths.

ms. All lattice beams were derived from the same laser operating at $\lambda=1064 \mathrm{~nm}$ and had an $1 / e^{2}$ waist of $100 \sim 200$ $\mu \mathrm{m}$. The lattice depth was calibrated with $1 \%$ accuracy by applying a $12.5 \mu$ s lattice laser pulse to a BEC and comparing the observed Kapitza-Dirac diffraction pattern of a BEC to theory. Fits of more than twenty diffraction patterns for the lattice depth from $<1 E_{R}$ to $40 E_{R}$ resulted in an accuracy of the calibration of $1 \%$.

For transport measurements, we moved an optical lattice [17, 22] which provides more flexibility to change the momentum than exciting a dipole oscillation by displacement of the BEC [23, 24]. A moving optical lattice with velocity $v=$ $\lambda \cdot \delta f / 2$ was created along the long axis of the BEC by introducing a small frequency detuning $\delta f$ between two counterpropagating lattice beams. If the velocity $v(t)$ changes slowly enough not to induce interband excitations, the initial Bloch state $|p=0\rangle$ of the condensate in the optical lattice adiabatically evolves into the current-carrying state $|p(t)=-m v(t)\rangle$ 
where $p$ is the quasi-momentum. For increasing lattice depth, the effective mass of atoms $m^{*}=\left[\partial^{2} E(p) / \partial p^{2}\right]^{-1}$ increases, and the group velocity $v_{g}=-\left(\mathrm{m} / \mathrm{m}^{*}\right) v(t)$ decreases. As a consequence, atoms prepared in a moving lattice with quasimomentum $p=-m v$ travel in the frame of the moving lattice with $v_{g}$ and in the lab frame with velocity $\Delta v=v+v_{g}=$ $\left(1-m / m^{*}\right) v$, which approaches $v$ in a deep lattice. Consequently, we observed that in a deep moving lattice atoms were dragged along to the edge of the trapping region limiting the experimental time scale to probe for dissipation. This became an issue for larger values of $p$ and was addressed by first ramping up the lattice with $p=0$ and then alternating the velocity of the moving lattice, thus performing a low-frequency AC transport measurement instead of DC.

We have used two sets of experimental procedures (Fig. 1), and our results were consistent for both. Close to the SF-MI phase transition, the lattice was increased to $V_{\text {latt }}$ with a fixed (and small) value of momentum $p$ (dahsed arrows in Fig. 1). After a variable hold time $t_{\text {hold }}$ at $V_{\text {latt }}$ the lattice was ramped down to zero, and the magnetic trap switched off. After $33 \mathrm{~ms}$ of ballistic expansion, the atoms were imaged and the condensate fraction was determined as a function of momentum. For smaller lattice depths, the lattice was ramped up with $p=0$ (Fig 1). Then a sinusoidal momentum modulation of the moving lattice with amplitude $p_{M}$ was applied by modulating the frequency detuning $\delta f$ between the counter-propagating lattice beams. The $10 \mathrm{~ms}$ period of this momentum modulation was slow enough to meet the adiabaticity condition, but fast enough to limit the displacement of the atomic cloud to less than a few $\mu \mathrm{m}$. Both the trapping potential and the optical lattice were then turned off suddenly. After $33 \mathrm{~ms}$ of ballistic expansion, the condensate fraction of the center peak of the superfluid interference pattern was recorded as a function of the momentum modulation amplitude $p_{M}$. Several cycles (typically, three to five) of the momentum modulation were applied to obtain a high contrast between the stable and dissipative regimes (Fig. 2 (a)).

Fig. 2 (a) shows how the transition between superfluid and dissipative currents became sharper with increasing number of cycles of the momentum modulation. The critical momentum was determined from a log-log plot of the condensate fraction as a function of momentum $p$ (Fig. 2 (c)). For low $p$, the condensate fraction is constant. For high $p$, the condensate fraction decreases. The intersection between two linear fit functions was taken as the critical momentum. Our result was found to be independent of the time period and number of cycles of the momentum modulation at a few percent level.

In the MI phase, stable superfluid flow is not possible and the critical momentum should vanish. However, using the procedure described above, we measured a small critical momentum of $0.02 p_{r}$ for lattice depths $V_{\text {latt }}=14,15,16 E_{R}$. Up to this momentum, the SF-MI phase transition remained reversible. We interpret the non-zero critical momentum as a finite-size effect. For our cloud size of $60 \mu \mathrm{m}$, the corresponding Heisenberg momentum uncertainty of $0.018 p_{r}$ agrees with our measured critical momentum. In cold atom

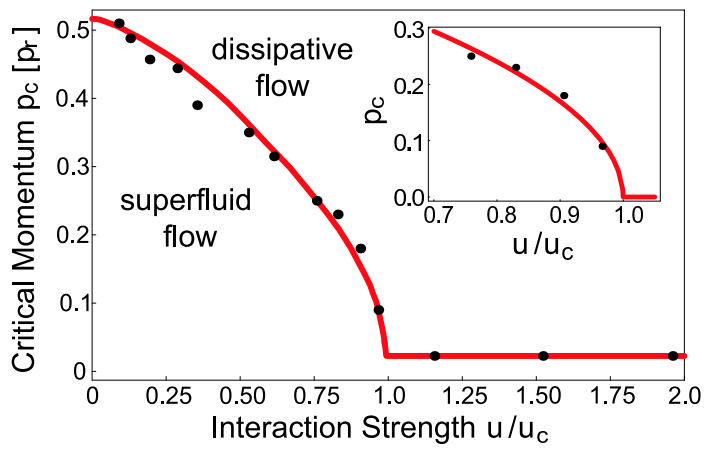

FIG. 3: Critical momentum for a condensate in a 3D lattice. The solid line shows the theoretical prediction for the superfluid region. The horizontal solid line is a fit to the data points in the MI phase. (Inset) Fit of critical momenta near the SF-MI phase transition.

experiments, some sloshing motion of the cloud in the trapping potential is unavoidable. The momentum uncertainty determined above indicates how much sloshing motion can be tolerated without affecting the observed phase transition.

The critical lattice depth for the SF-MI phase transition can be determined as the point where the critical momentum vanishes. Using the predicted functional form [9] of the approach towards zero, $p_{c} \propto \sqrt{1-u / u_{c}}$, as a fit function for the data points close to the SF-MI phase transition (the data points shown in the inset of Fig. 3) we determined the critical value $u_{c}=34.2( \pm 2.0)$ corresponding to a lattice depth of $13.5( \pm 0.2) E_{R}$ [29]. Our result agrees with the mean field theory prediction $u_{c}=5.8 \times 6=34.8$ for $N=1$ SFMI phase transition [1] and deviates by $2 \sigma$ from the predictions of $u_{c}=29.34(2)$ of quantum Monte Carlo(QMC) simulation [25, 26], which includes corrections beyond the mean field theory. This demonstrates that our method has the precision to identify non-mean field corrections. However, to turn precision into accuracy, experiments or QMC simulations $[20,25,26]$ have to address corrections due to finite size, finite temperature, and finite time to probe the onset of the instability [26]. In our experiment, these corrections seemed to be small, but have not been characterized at the level of $1 \%$ in lattice depth.

The mean-field prediction for stable superfluid flow in 1D is similar to that for the 3D system [9]. However, it is well known that fluctuations play a much more important role in 1D. For studying a 1D system, we prepared an array of onedimensional gas tubes by ramping two pairs of optical lattice beams up to lattice depths of $V_{x}=V_{y}=30 E_{R}$ suppressing hopping between the tubes. After a hold time of $10 \mathrm{~ms}$, a moving optical lattice was ramped up along the z-axis. As in our 3D experiment, a momentum modulation was applied, after which the moving optical lattice was ramped down to zero, followed by the other two optical lattices. The condensate fraction was determined after $33 \mathrm{~ms}$ of ballistic expansion as a function of the momentum modulation amplitude. The critical momentum, where the onset of dissipation begins, was identified from a log-log plot as in the $3 \mathrm{D}$ case. Since the 
(a)

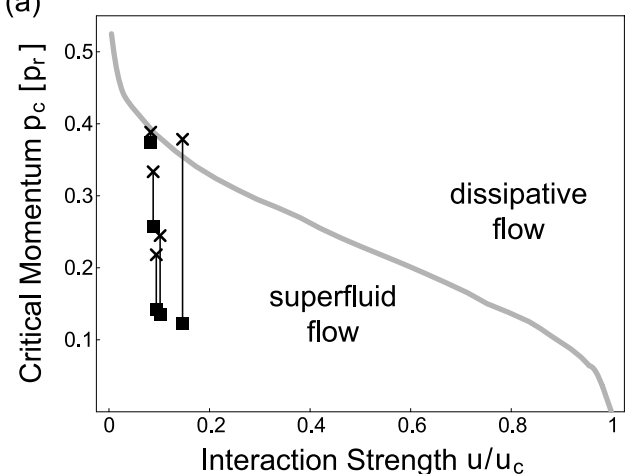

(b)
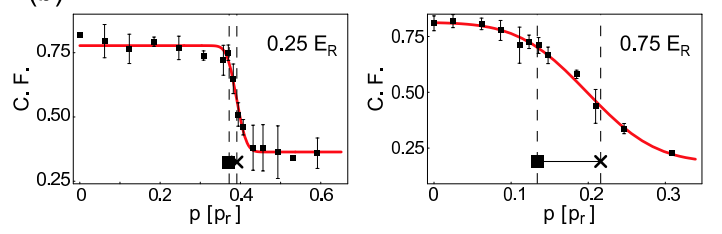

FIG. 4: Critical momentum for a 1D gas in an optical lattice. (a) The grey line indicates the mean-field theory prediction. The interaction strengths are normalized by the mean-field prediction for $u_{c}=5.8 \times 2[1,4]$. Squares(crosses) represent the measured critical momentum (the center of the transition). Measurements were taken at lattice depths of $0.25,0.50,0.75,1.0,2.0 E_{R}$. The lines between crosses and squares indicate the width of the transition region. (b) Condensate fraction measured at $0.25 E_{R}$ (left) and 0.75 $E_{R}$ (right).The data were fitted with an error function. Squares (the critical momentum) and crosses (the center of the transition) are indicated on the plots.

transitions became very broad, we characterized them by an error function fit, with the center of the fitted error function taken as the center of the transition (Fig. 4).

In the $1 \mathrm{D}$ system, at a very shallow lattice depth of 0.25 $E_{R}$ (corresponding to $u / u_{c}=0.08$ ) a sharp transition was observed, and the measured critical momentum agreed very well with the prediction [9, 27] of a critical momentum of $0.39 p_{r}$. However, a slight increase of the interaction strength (to $u / u_{c}=0.09$ at a lattice depth of $0.5 E_{R}$ ) led to a significant decrease of the critical momentum as well as a dramatic broadening of the transition as shown in Fig. 4 For lattice depths larger than $2 E_{R}$, the transition became very broad and showed complex behavior, and we could not obtain quantitative fits. Our results show a significant deviation from the mean-field theory predictions and are in agreement with previous experiments [24] where damped dipole oscillations of a 1D Bose gas in an optical lattice were observed, and the damping grew rapidly even at very shallow lattice depths around $0.25 E_{R}$.

The observed broadening of the transition confirms theoretical studies which emphasize the importance of quantum fluctuations in the 1D system. Quantum tunneling out of metastable states which are ignored in the mean-field description can lead to a decay of the superfluid current at very low momenta [27]. In addition to quantum fluctuations, thermal fluctuations provide a mechanism for current decay [27]. In our experiment, we used a "pure" BEC without a discernible thermal component. The close agreement with $T=0$ predictions indicates that thermal fluctuations were not dominant.

In conclusion we have used transport studies to connect a well-known dynamical instability for weakly interacting bosons with the equilibrium superfluid to Mott insulator transition. A comparison of 3D and 1D systems confirms the applicability of a mean-field description in 3D and the crucial importance of fluctuations in 1D. The disappearance of superfluid currents at the SF-MI phase transition precisely located the phase transition. Our results illustrate the control and precision of condensed matter physics experiments done with ultracold atoms and their suitability to test many-body theories.

This work was funded by NSF through the grant for CUA. L.G.M. acknowledges support from Coordenacao de Aperfeicoamento de Pessoal de Nivel Superior. We thank E. Demler and A. Polkovnikov for insightful discussions, and David Weld for a critical reading of the manuscript.

* current address: JILA, Boulder, Colorado 80309, USA.

$\dagger$ permanent address: Instituto de Fisica de São Carlos, University of São Paulo, São Carlos, 13560-970, SP, Brazil

[1] D. Jaksch, C. Bruder, J. I. Cirac, C. W. Gardiner, and P. Zoller, Phys. Rev. Lett. 81, 3108 (1998).

[2] C. Orzel, A. K. Tuchman, M. L. Fenselau, M. Yasuda, and M. A. Kasevich, Science 291, 2386 (2002).

[3] M. Greiner, O. Mandel, T. Esslinger, T. W. Hänsch, and I. Bloch, Nature 415, 39 (2002).

[4] T. Stöferle, H. Moritz, C. Schori, M. Kohl, and T. Esslinger, Phys. Rev. Lett. 92, 130403 (2004).

[5] S. Fölling, F. Gerbier, A. Widera, O. Mandel, T. Gericke, and I. Bloch, Nature 434, 481 (2005).

[6] F. Gerbier, A. Widera, S. Fölling, O. Mandel, T. Gericke, and I. Bloch, Phys. Rev. Lett. 95, 050404 (2005).

[7] G. K. Campbell, J. Mun, M. Boyd, P. Medley, A. E. Leanhardt, L. G. Marcassa, D. E. Prichard, and W. Ketterle, Science 313, 649 (2006).

[8] S. Fölling, A. Widera, T. Muller, F. Gerbier, and I. Bloch, Phys. Rev. Lett. 97, 060403 (2006).

[9] E. Altman, A. Polkovnikov, E. Demler, B. I. Halperin, and M. D. Lukin, Phys. Rev. Lett. 95, 020402 (2005).

[10] M. P. A. Fisher, P. B. Weichman, G. Grinstein, and D. S. Fisher, Phys. Rev. B 40, 546 (1989).

[11] W. Krauth, M. Caffarel, and J.-P. Bouchaud, Phys. Rev. B 45, 3137 (1992).

[12] J. K. Freericks and H. Monien, Europhys. Lett. 26, 545 (1994).

[13] B. Wu and Q. Niu, Phys. Rev. A 64, 061603(R) (2001).

[14] G. K. Campbell, J. Mun, M. Boyd, E. W. Streed, W. Ketterle, and D. E. Pritchard, Phys. Rev. Lett. 96, 020406 (2006).

[15] K. M. Hilligsøe and K. Mølmer, Phys. Rev. A 71, 041602(R) (2005).

[16] M. Modugno, C. Tozzo, and F. Dalfovo, Phys. Rev. A 70, 043625 (2004).

[17] L. Fallani, L. D. Sarlo, J. E. Lye, M. Modugno, R.Saers, and M. Inguscio, Phys. Rev. Lett. 93, 140406 (2004).

[18] L. D. Sarlo, L. Fallani, J. E. Lye, M. Modugno, R. Saers, C. Fort, and M. Inguscio, Phys. Rev. A 72, 013603 (2005).

[19] B. Wu and J. Shi, cond-matt/0607098 (2006). 
[20] S. Wessel, F. Alet, M. Tryoer, and G. G. Batrouni, Phys. Rev. A 70, 053615 (2004).

[21] V. A. Kashurnikov, N. V. Prokof'ev, and B. V. Svistunov, Phys. Rev. A 66, 031601 (2002).

[22] M. Ben Dahan, E. Peik, J. Reichel, Y. Castin, and C. Salomon, Phys. Rev. Lett. 76, 4508 (1996).

[23] F. S. Cataliotti, S. Burger, C. Fort, P. Maddaloni, F. Minardi, A. Trombettoni, A. Smerzi, and M. Inguscio, Science 293, 843 (2001).

[24] C. D. Fertig, K. M. O’Hara, J. H. Huckans, S. L. Rolston, W. D. Phillips, and J. V. Porto, Phys. Rev. Lett. 94, 120403 (2005).

[25] J. K. Freericks and H. Monien, Phys. Rev. B 53, 2691 (1996).
[26] B. Capogrosso-Sansone, N. V. Prokof'ev, and B. V. Svistunov, cond-matt/0701178 (2007).

[27] A. Polkovnikov, E. Altman, E. Demler, B. Halperin, and M. D. Lukin, Phys. Rev. A 71, 063613 (2005).

[28] D. M. Harber, H. J. Lewandowski, J. M. McGuirk, and E. A. Cornell, Phys. Rev. A 66, 053616 (2002).

[29] The lattice depth was converted to dimensionless interaction energy $u$ following the method in ref. [1] using Wannier function truncated at the 5th excited Bloch band and a s-wave scattering length $a=100.44 a_{0}$ [28] ( $a_{0}$ : Bohr radius). 